\title{
Highly efficient fluorescence quenching with chemically exfoliated reduced graphene oxide
}

Shubhda Srivastava, Thanikachalam Devarajan Senguttuvan, and Bipin Kumar Gupta

Citation: Journal of Vacuum Science \& Technology B 36, 04G104 (2018); doi: 10.1116/1.5026170

View online: https://doi.org/10.1116/1.5026170

View Table of Contents: http://avs.scitation.org/toc/jvb/36/4

Published by the American Vacuum Society

\section{Articles you may be interested in}

Preparation and characterization of $\mathrm{Al}_{2} \mathrm{O}_{3}$ film deposited by $\mathrm{RF}$ sputtering and plasma enhanced atomic layer deposition

Journal of Vacuum Science \& Technology B 36, 04 G101 (2018); 10.1116/1.5023591

Near-infrared light absorption enhancement in Ge nanostructures prepared by nanosphere lithography Journal of Vacuum Science \& Technology B 36, 041601 (2018); 10.1116/1.5029435

Dry etch damage in n-type crystalline silicon wafers assessed by deep-level transient spectroscopy and minority carrier lifetime

Journal of Vacuum Science \& Technology B 36, 041201 (2018); 10.1116/1.5026529

Beyond equilibrium thermodynamics in the low temperature plasma processor Journal of Vacuum Science \& Technology B 36, 048501 (2018); 10.1116/1.5022470

Enhanced temperature and light stability of amorphous indium-gallium-zinc oxide thin film transistors by interface nitrogen doping

Journal of Vacuum Science \& Technology B 36, 040601 (2018); 10.1116/1.5031471

High-performance field emission device utilizing vertically aligned carbon nanotubes-based pillar architectures AIP Advances 8, 015117 (2018); 10.1063/1.5004769

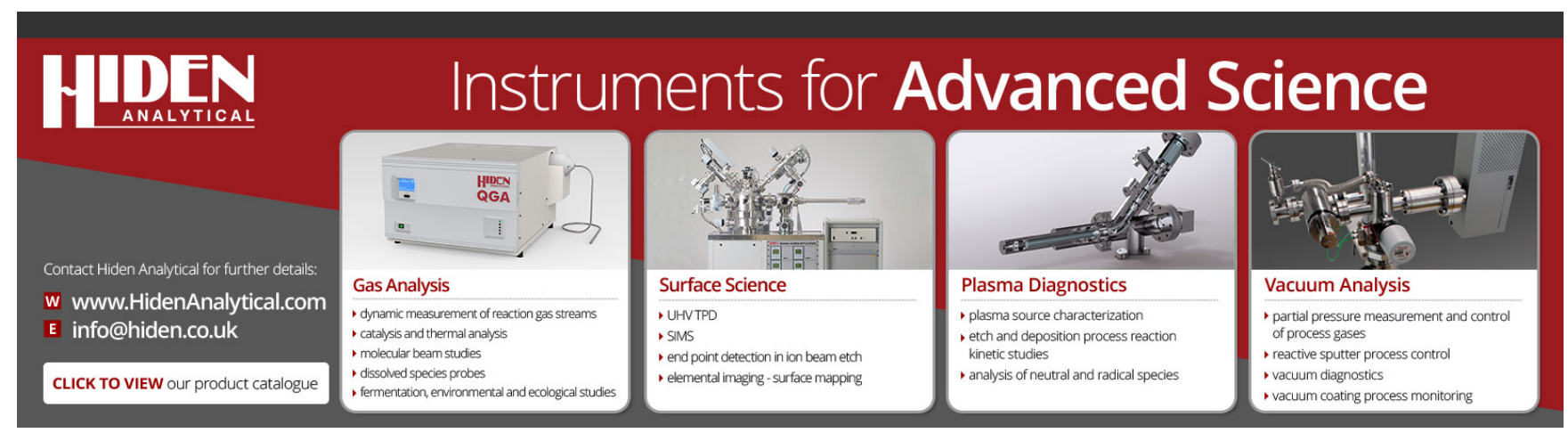




\title{
Highly efficient fluorescence quenching with chemically exfoliated reduced graphene oxide
}

\author{
Shubhda Srivastava ${ }^{\text {a) }}$ \\ Academy of Scientific and Innovative Research (AcSIR), CSIR-NPL Campus, New Delhi 110012, India \\ Thanikachalam Devarajan Senguttuvan and Bipin Kumar Gupta ${ }^{\text {b) }}$ \\ CSIR-National Physical Laboratory, New Delhi 110012, India
}

(Received 16 February 2018; accepted 21 May 2018; published 7 June 2018)

\begin{abstract}
Fluorescence quenching is a valuable tool to study many macromolecular assemblies. It is well-known that graphitic systems such as the reduced graphene oxide (rGO) can strongly quench the emission of dye molecules through energy transfer. In this work, the authors demonstrate comparative study of fluorescence quenching between different graphitic system such as rGO, graphene oxide (GO), and graphite, which is not reported so far. As compared to graphite and GO sheets, rGO shows more quenching capability because of more surface area and efficient $\pi$ - $\pi$ stacking. The fluorescence quenching has been performed using Rhodamine B dye. The scanning electron microscopic micrographs revealed that Rhodamine B dye is uniformly absorbed on rGO. The fluorescence quenching has been done with the fixed concentration of Rhodamine organic dye with all three forms of carbon. The spectroscopic results exhibit that the chemically exfoliated rGO was almost 16 times better than graphite and GO. The obtained results suggest that rGO can be used for better quenching capability in biosensing applications. Published by the AVS. https://doi.org/10.1116/1.5026170
\end{abstract}

\section{INTRODUCTION}

Graphene, one atom thick layer of $\mathrm{sp}^{2}$ carbon atoms, has attracted enormous interests for various applications because of its outstanding optical, thermal, and electrical properties. $^{1-4}$ In some optical sensing applications, graphene, specially graphene oxide (GO)/reduced graphene oxide (rGO), can be efficiently used as a basis for biosensors through the energy transfer mechanism, and because of its transparent semimetal nature, graphene is recently the most unique interface for the fluorescence energy transfer. Fluorescence quenching is a key technique to get information about the dynamic changes of proteins in macromolecular systems. 5,6 In this quenching phenomena, the emission of a fluorophore is disturbed by a quencher molecule through fluorescence (or Forster) resonance energy transfer. Very interestingly, although GO and rGO are themselves fluorescent, they can also quench the fluorescence and this contradictory property could be the manifestation of the heterogeneous chemical, atomic, and electronic structures of $\mathrm{GO} / \mathrm{rGO}$. Applications have been seen where GO has been used for its fluorescence quenching capability. ${ }^{7}$ Also, graphene has been used as a substrate for studying fluorescence suppression in Raman resonance spectroscopy. ${ }^{8}$ The $\mathrm{sp}^{2}$ carbon domains in GO and rGO allow it to quench fluorescence of dyes, ${ }^{9,10}$ quantum dots, ${ }^{11}$ and conjugated polymers. ${ }^{12,13}$ After reduction, the quenching efficiency of GO increases significantly ${ }^{10}$ and pristine graphene can quench the fluorescence of dyes up to 3 orders of magnitude. ${ }^{10}$ Studies have shown that this fluorescence quenching comes from the fluorescence resonance energy transfer between the fluorescent species and GO/rGO. ${ }^{10,11,13}$

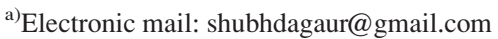

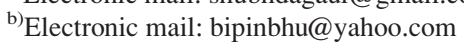

The presence of ionic groups such as $\mathrm{O}^{-}$and $\mathrm{COO}^{-}$in $\mathrm{GO} / \mathrm{rGO}$ makes it possible to interact electrostatically with charged proteins and DNA, and the aromatic scaffold in it provides a platform for $\pi-\pi$ stacking and quenching of dyes. The importance of the area is directly associated with biosensing applications which is based on fluorescence quenching in rGO. For example, such applications can be realized in single-stranded DNA sensing through GO. ${ }^{14}$ Hence, fluorescence quenching has been used as the basis of $\mathrm{GO} / \mathrm{rGO}$ optical sensors for sensing DNA and biomolecules. ${ }^{14,15}$ In the present study, Rhodamine dye has been taken because of the easy availability of the Rhodamine B dye, which is used as calibration dye for photoluminescence (PL) setup. Other dyes like fluorescein, 4-(dicyanomethylene)-2-methyl-6-(4dimethylaminostyryl)-4H-pyran, and 2,5-bis(5-tert-butyl-2benzoxazolyl)thiophene can also be used in such kinds of studies.

Various forms of graphene, especially graphene oxide and exfoliated graphene, have been used for quenching applications, but the quantitative analysis of quenching efficiency of GO and $\mathrm{rGO}$ has not been reported yet. In this study, chemically exfoliated rGO has been shown a very efficient quencher for fluorescence molecules as compared to graphite and GO. Also, a novel synthesis method for GO and further reduction to $\mathrm{rGO}$ has been presented.

\section{EXPERIMENT}

\section{A. Synthesis of graphene oxide}

GO was prepared by the oxidation of graphite (500 mesh powder) using the improved Hummer's method. ${ }^{16}$ For this, $3.0 \mathrm{~g}$ of graphite powder was added to a 9:1 mixture of conc. $\mathrm{H}_{2} \mathrm{SO}_{4} / \mathrm{H}_{3} \mathrm{PO}_{4}(360 / 40 \mathrm{ml})$ and stirred. $18.0 \mathrm{~g}$ of $\mathrm{KMnO}_{4}$ was slowly added to the suspension while stirring. Then, the resultant mixture was heated at $60^{\circ} \mathrm{C}$ and stirred for another 
$12 \mathrm{~h}$. After cooling down to room temperature, $400 \mathrm{ml}$ deionized water (DI) water was added followed by a gradual addition of $3 \mathrm{ml} \mathrm{H}_{2} \mathrm{O}_{2}$ (30\%). The resultant material was washed with DI water and $35 \% \mathrm{HCl}$ mixture (2:1) and filtered in a vacuum filtration setup using a G4 polymer filter. Then, the solution was ultrasonicated for $8 \mathrm{~h}$ in ethanol in order to obtain GO.

\section{B. Synthesis of reduced graphene oxide}

Reduced graphene oxide was obtained from thermal reduction of GO. The as prepared GO powder was taken in an alumina boat and kept in a quartz tube fitted to a high temperature furnace. The GO powder was heated at $800^{\circ} \mathrm{C}$ for $2 \mathrm{~h}$ under 85:15 vol. \% gas flow of $\mathrm{Ar}$ and $\mathrm{H}_{2}{ }^{7}$ This treatment resulted in a fluffy, very light weight and highly exfoliated rGO.

\section{Sample preparation}

Rhodamine B with emission at $574 \mathrm{~nm}$ was taken as organic fluorophore for fluorescence quenching study, and $10 \mathrm{ppm}$ solution was prepared by mixing the Rhodamine B in DI water. For a comparative study, a fixed amount of $2 \mathrm{mg}$ of graphite, GO, and rGO was dispersed in $20-20 \mathrm{ml}$ of DI water by ultrasonication for around $30 \mathrm{~min}$ to make a clear dispersion. Now, each dispersion was mixed with $15 \mathrm{ml}$ of Rhodamine B solution and was kept for 5 min before recording their PL spectra. Figure 3(b) shows the as prepared solutions.

\section{Fluorescence quenching study}

The excitation spectrum of pure Rhodamine was taken before performing the quenching study. After this, the photoluminescence spectra of all three (graphite, GO, and rGO) dispersions mixed with Rhodamine solution were recorded. For the ease of comparison, all the parameters of spectrometer like concentration of dye in all variants of carbon as well as pure dye in DI water, slit width of excitation and emission window, temperature, and volume of the solution in all cases.

\section{CHARACTERIZATIONS}

Raman studies of the samples were done using Renishaw inVia Raman spectroscopy, UK, with an excitation laser source of $514 \mathrm{~nm}$. Field emission scanning electron microscopy was done using Auriga, Zeiss instrument. Photoluminescence spectra of the samples were recorded using FLSP 900 (Edinburg) where the xenon flash lamp acts as a source of excitation.

\section{RESULTS AND DISCUSSIONS}

Prior to the quenching experiment, the surface morphology of graphite, GO, and rGO was investigated using a field emission scanning electron microscope (FESEM). Figures 1(a)-1(c) show the typical FESEM images of graphite, GO and rGO, respectively. The high magnification FESEM image [Fig. 1(d)] clearly showed the few layered rGO sheets. Raman studies of graphite as well as GO and rGO were done to ensure the quality.
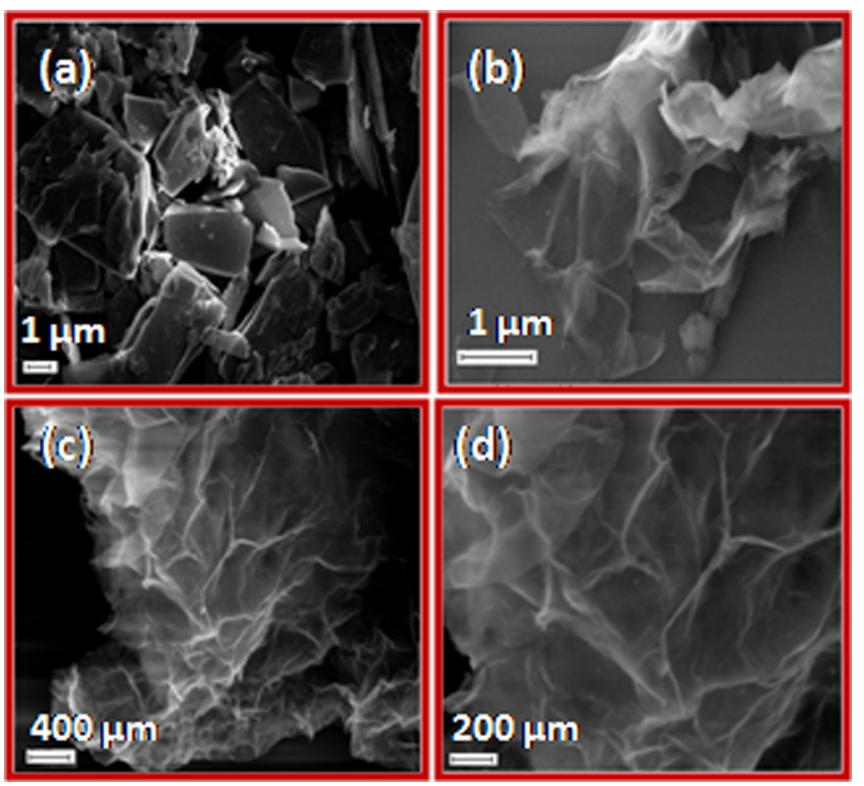

FIG. 1. (Color online) FESEM images of (a) graphite powder, (b) GO sheets, (c) rGO sheets, and (d) high magnification image of rGO sheets.

It is well studied that all graphitic systems ${ }^{15,17,18}$ can strongly quench the emission of fluorescent dye molecules through energy transfer. The PL excitation spectrum of Rhodamine B at an emission wavelength of $574 \mathrm{~nm}$ is illustrated in Fig. 2(a), which is the observation for standard commercially available Rhodamine solution. The PL emission spectra of pure Rhodamine B, graphite + Rhodamine B, $\mathrm{GO}+$ Rhodamine $\mathrm{B}$, and $\mathrm{rGO}+$ Rhodamine $\mathrm{B}$ at the excitation wavelength $558 \mathrm{~nm}$ are shown in Fig. 2(b). Figure 2(b) shows that the PL emission of Rhodamine B solution can be quenched significantly with all three graphitic systems. However, rGO shows highest fluorescence quenching capability as compared to other graphitic systems used. The PL emission spectrum of Rhodamine B shows a broad emission band peak at $574 \mathrm{~nm}$. Figure 2(b) reveals that the emission intensity of Rhodamine B is of the order of $10^{5}$. When the PL spectra of the dye with graphite, GO and rGO were recorded, it was interestingly observed that in the case of graphite and GO, the PL quenching was of the order of $10^{4}$, while in the case of chemically exfoliated rGO, it was almost completely quenched, as shown in Fig. 2(b). The quenching capability of rGO is more as compared to GO and graphite because rGO provides more surface area as compared to graphite and GO. Further, in comparison between GO and rGO, GO has more terminal bonds in terms of functional groups (hydroxyl, epoxy, carbonyl, and carboxyl groups) which is almost reduced in the case of rGO (hydroxyl and carboxyl groups). Figure 2(c) shows the PL quenching capability of all three on a logarithmic scale to better visualize the quenching behavior. Figure 3(a) illustrates the schematic of how the dye molecules are attached with the graphene sheets. Figure 3(b) shows that the quenching effect increases from graphite to GO and then to $\mathrm{rGO}$ with the change in the dye solution color. Also, it could be visibly evidenced through naked eye from Figs. 3(b) and 3(c) that the pink 

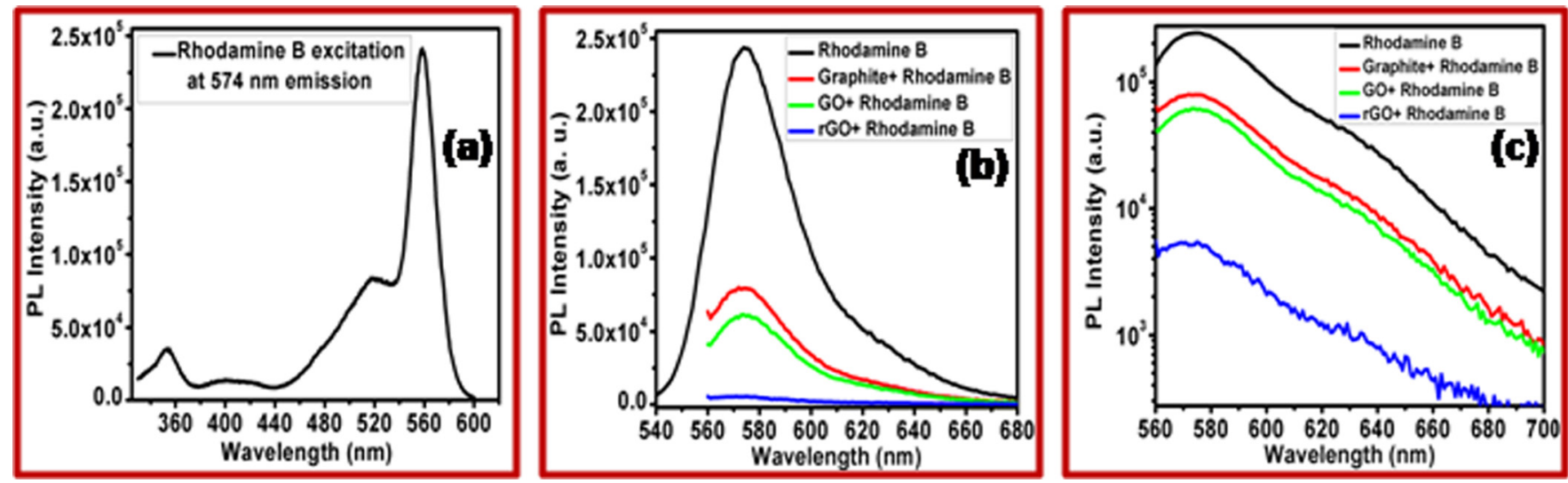

FIG. 2. (Color online) (a) Excitation spectrum of pristine Rhodamine B with $574 \mathrm{~nm}$ emission at room temperature, (b) photoluminescence quenching emission spectra of pristine Rhodamine B, graphite + Rhodamine B, GO + Rhodamine B, and $\mathrm{rGO}+$ Rhodamine $\mathrm{B}$, and (c) photoluminescence quenching emission spectra of pristine Rhodamine B, graphite + Rhodamine B, GO + Rhodamine B, and rGO + Rhodamine B on a logarithmic scale.

color of Rhodamine dye in rGO + Rhodamine mixture has totally vanished, which confirms the fluorescence quenching of the dye after adsorption with the rGO sheets through $\pi-\pi$ stacking. This strongly supports our results.

The effect of concentration on fluorescence quenching capability of rGO was studied. For this study, various amounts of rGO were mixed in a fixed amount of dye and the PL spectra were recorded. Figure S2 shows a bar chart of increasing capability of fluorescence quenching with the increase in the amount of rGO. ${ }^{21}$ It is seen from Fig. S2 that after a fixed amount of $\mathrm{rGO}(0.1 \mathrm{mg})$ in mixed solution, the further increase in rGO does not make any major increase in the quenching capability of rGO.

Further, to visualize the quenching process with $\mathrm{rGO}$, the FESEM was performed for Rhodamine $\mathrm{B}+\mathrm{rGO}$ sample. Figure 4(a) shows the FESEM images of Rhodamine $\mathrm{B}+\mathrm{rGO}$. The two shades of rGO sheets show the attachment of dye molecules at some places (dark portions) and the pure rGO sheets at other places (light portions). Figure 4(b) shows
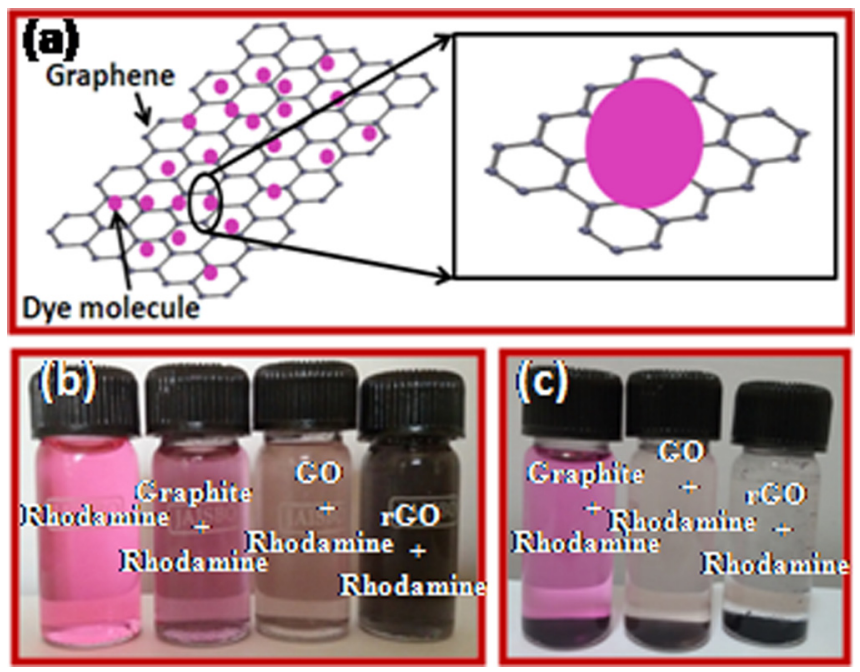

FIG. 3. (Color online) (a) Schematic diagram showing dye molecules attached with graphene. (b) Photograph showing the quenching effects of graphite, GO, and rGO. (c) Fluorescence quenched mixture solution colors after $1 \mathrm{~h}$. the high magnification image of rGO sheet in which dye molecules are attached. The two portions of the sheet surface have been marked as pure rGO sheets and Rhodamine + rGO sheets in Fig. 4(b).

Raman studies of graphite powder, GO, and rGO were performed to ensure the quality of materials. It is well studied that Raman spectra of natural graphite has two main bands, i.e., $\mathrm{G}$ band at $1587 \mathrm{~cm}^{-1}$, and 2D band at $2700 \mathrm{~cm}^{-1}$, and also a third very weak $D$ band at $1353 \mathrm{~cm}^{-1}$. The $\mathrm{G}$ band is an in-plane vibrational mode involving the $\mathrm{sp}^{2}$ hybridized carbon atoms and $\mathrm{D}$ band represents ring breathing mode from $\mathrm{sp}^{2}$ carbon rings and requires a defect for its activation. It is also known as a disorder or defect band. The 2D band is the second order of the $\mathrm{D}$ band and sometimes referred to as an overtone of the D band. It is the result of a two phonon lattice vibrational process but does not need any defect to be activated. GO exhibits D band at $1353 \mathrm{~cm}^{-1}$ and $\mathrm{G}$ band at $1597 \mathrm{~cm}^{-1}$ with a blue shift in frequency as compared to graphite and this shift in the frequency of $\mathrm{G}$ band could be because of the decrease in the size of in-plane $\mathrm{sp}^{2}$ domains during oxidation of graphite. ${ }^{19,20}$ Raman spectra of graphite, GO, and rGO are shown in Fig. 5(a). For GO, the G band was located at $\sim 1602 \mathrm{~cm}^{-1}$, while for rGO this band was shifted to $\sim 1587 \mathrm{~cm}^{-1}$. This indicated the reduction of GO
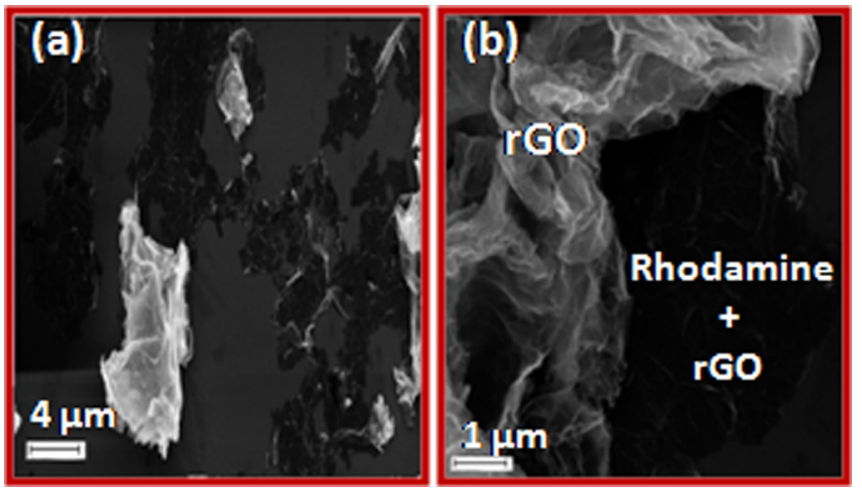

FIG. 4. (Color online) FESEM images of (a) rGO sheets mixed with Rhodamine B and (b) high magnification image of rGO sheets mixed with Rhodamine B with pure rGO sheets and Rhodamine + rGO portions marked. 

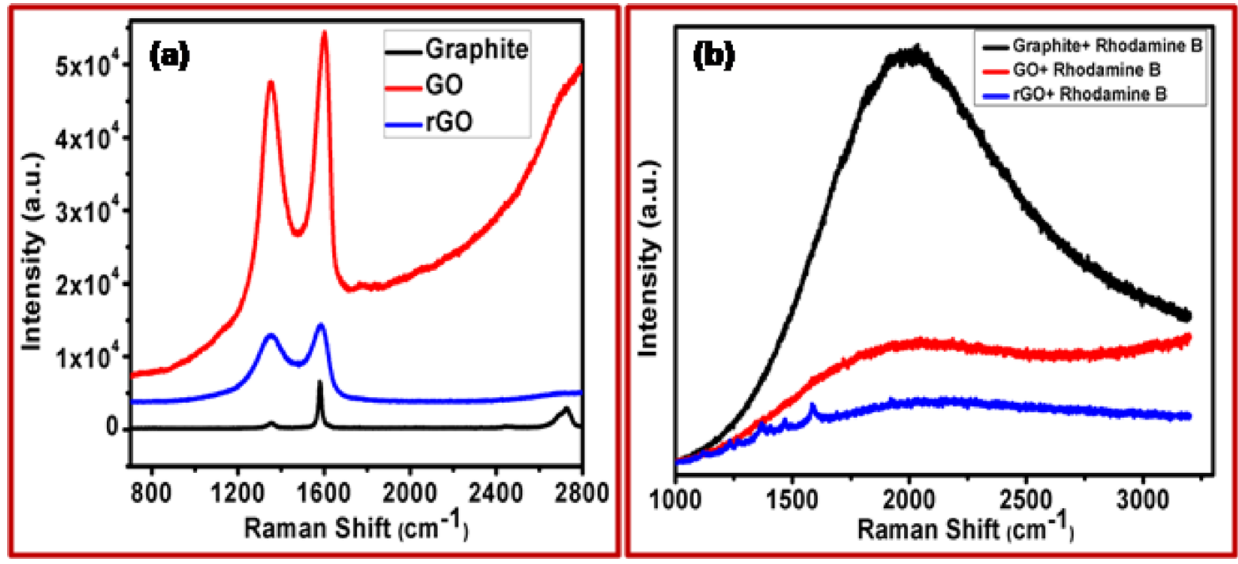

FIG. 5. (Color online) Raman spectra of (a) graphite, GO, and rGO, (b) (graphite + Rhodamine B), (GO + Rhodamine B), and (rGO + Rhodamine B).

to $\mathrm{rGO}$. The $\mathrm{D}$ bands for $\mathrm{GO}$ and $\mathrm{rGO}$ were located at $\sim 1351$ and $\sim 1344 \mathrm{~cm}^{-1}$, respectively. The intensity ratio of these bands $\left(\mathrm{I}_{\mathrm{D}} / \mathrm{I}_{\mathrm{G}}\right)$ for rGO $(0.90)$ was higher than for $\mathrm{GO}$ (0.88).

To further support our fluorescence quenching results, the Raman spectroscopy of pure Rhodamine B solution and mixed solutions of graphite, GO, and rGO with Rhodamine $\mathrm{B}$ was done to investigate the change in the peak positions and their intensities. Figure 5(b) shows the Raman spectra of mixed solutions of (graphite + Rhodamine B), (GO + Rhodamine B), and (rGO + Rhodamine B). It can be easily seen that for graphite, the fluorescence in mixed solution is almost the same as that of Rhodamine B. In the case of $\mathrm{GO}$, fluorescence has been reduced as compared to the pure Rhodamine $\mathrm{B}$, and with rGO, the fluorescence has been reduced greatly. The Raman spectra for Rhodamine B are shown in Fig. S1. ${ }^{21}$

\section{SUMMARY AND CONCLUSIONS}

The results presented in this paper show that chemically exfoliated reduced graphene oxide can efficiently quench the fluorescence of aromatic fluorescence dyes as compared to graphite and graphene oxide. A comparative study of quenching capability of all three, graphite, GO, and $\mathrm{rGO}$, has been done successfully. All the results follow the trend of increasing capability of fluorescence quenching from graphite to GO and to rGO. On the basis of the present study, it is possible that the synthesized and chemically exfoliated rGO can be efficiently used in biosensing applications.

\section{ACKNOWLEDGMENTS}

The authors are grateful to the Director of CSIR-National Physical Laboratory, New Delhi, for encouragement and his keen interest in this work. The authors are also thankful to $\mathrm{S}$. Sharma for performing the Raman Spectroscopy of the samples and V. N. Singh for providing the FESEM measurements. Shubhda Srivastava would like to thank the Department of Science and Technology (Ministry of Science and Technology) for financial assistance under the Women Scientist scheme (No. SR/WOS-A/ET-49/2013).

${ }^{1}$ A. K. Geim and K. S. Novoselov, Nat. Mater. 6, 183 (2007).

${ }^{2}$ A. K. Geim, Science 324, 1530 (2009).

${ }^{3}$ Y. Zhang, J. W. Tan, H. L. Tormer, and P. Kim, Nature 438, 201 (2005).

${ }^{4}$ K. S. Novoselov, A. K. Geim, S. V. Morozov, D. Jiang, Y. Zhang, S. V. Dubonos, I. V. Grigorieva, and A. A. Firsov, Science 306, 666 (2004).

${ }^{5}$ L. Matyusa, J. Szollosia, and A. J. Jeneia, Photochem. Photobiol., B 83, 223 (2006).

${ }^{6}$ X. Zhuang, T. Ha, H. D. Kim, T. Centner, S. Labeit, and S. Chu, Proc. Natl. Acad. Sci. U. S. A. 9, 14241 (2000).

${ }^{7}$ B. K. Gupta, P. Thanikaivelan, T. N. Narayanan, L. Song, W. Gao, T. Hayashi et al., Nano Lett. 11, 5227 (2011).

${ }^{8}$ L. Xie, X. Ling, Y. Fang, J. Zhang, and Z. Liu, J. Am. Chem. Soc. 131, 9890 (2009).

${ }^{9}$ E. Treossi et al., J. Am. Chem. Soc. 131, 15576 (2009).

${ }^{10}$ J. Kim, L. J. Cote, F. Kim, and J. Huang, J. Am. Chem. Soc. 132, 260 (2010).

${ }^{11}$ H. Dong, W. Gao, F. Yan, H. Ji, and H. Ju, Anal. Chem. 82, 5511 (2010).

${ }^{12}$ Z. Liu, Q. Liu, Y. Huang, Y. Ma, S. Yin, X. Zhang, W. Sun, and Y. Chen, Adv. Mater. 20, 3924 (2008).

${ }^{13}$ Y. Wang, D. Kurunthu, G. W. Scott, and C. J. Bardeen, J. Phys. Chem. C 114, 4153 (2010).

${ }^{14}$ S. He et al., Adv. Funct. Mater. 20, 453 (2010).

${ }^{15}$ C.-H. Lu, H.-H. Yang, C.-L. Zhu, X. Chen, and G.-N. Chen, Angew. Chem. Int. Ed. 48, 4785 (2009).

${ }^{16}$ D. C. Marcano, D. V. Kosynkin, J. M. Berlin, A. Sinitskii, Z. Sun, A. Slesarev, L. B. Alemany, W. Lu, and J. M. Tour, ACS Nano 4, 4806 (2010).

${ }^{17}$ N. Nakayama-Ratchford, S. Bangsaruntip, X. Sun, K. Welsher, and H. J. Dai, Am. Chem. Soc. 129, 2448 (2007).

${ }^{18}$ M. R. Kagan and R. L. McCreery, Anal. Chem. 66, 4159 (1994).

${ }^{19}$ S. S. J. Aravind, V. Eswaraiah, and S. Ramaprabhu, J. Mater. Chem. 21, 15179 (2011).

${ }^{20}$ A. Ferrari and J. Robertson, Phys. Rev. B 61, 14095 (2000).

${ }^{21}$ See supplementary material at https://doi.org/10.1116/1.5026170 for Raman spectra of pure Rhodamine B dye and bar chart representation of fluorescence quenching capability of different concentrations of rGO in fixed Rhodamine solution. 